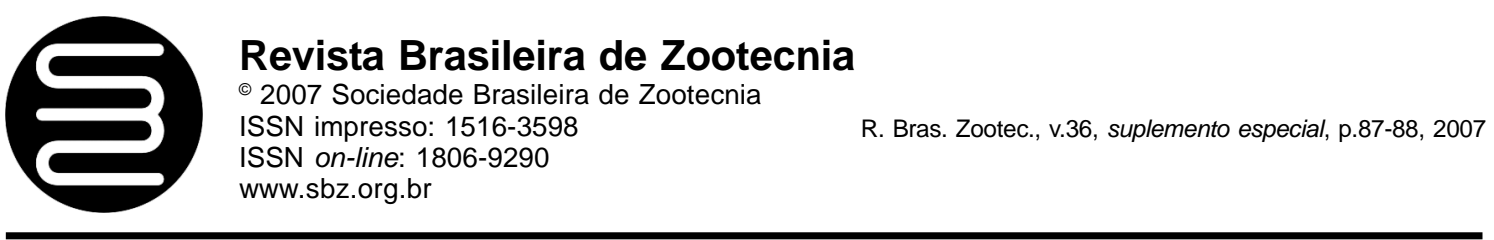

\title{
Non-invasive endocrine monitoring using fecal steroid analysis: opportunities and challenges
}

\section{Franz Schwarzenberger}

Dept. of Natural Sciences - Biochemistry University of Veterinary Medicine Veterinarplatz 1 A-1210 Vienna Austria Fax: +43-125077 4190. Franz.Schwarzenberger@vu-wein.ac.at

\begin{abstract}
During the past two decades, techniques for the non-invasive analysis of fecal steroid metabolites (estrogen, androgen, progestagen and glucocorticoid metabolites) have been developed. These techniques have been used for research with mammals, birds, reptiles, and to a lesser extent in fish; species studied included domestic and laboratory animals, as well as captive and free-ranging wildlife. The techniques now are widely accepted, and in many studies in which either blood sampling on a regular basis was difficult or not possible, i.e. in research on wildlife, very stress prone species or animals of small size, it has become a substitute for analyzing steroid hormones in serum/plasma.
\end{abstract}

Key Words: adrenal steroid hormones, assay validation, faecal steroid analysis, non-invasive, reproductive steroid hormones, steroid metabolism

Fecal steroid analysis is non-invasive to the investigated subjects and as such it does not introduce variables that may alter results. Further it has the advantage of presenting a more dampened hormone profile over time with less interfering from daily rhythm and acute stress. Animal taxa for which fecal steroid analysis provided thourough and detailed knowledge on the endocrine physiology summarized in recent reviews include primates (Shimizu, 2005), cats (Brown, 2006) and rhinoceroses (Roth, 2006). In addition, analysis in certain animal taxa like primates, marsupials, pilosa, rodentia, proboscidea, perissodactyla, artiodactyla and carnivora has been summerized by i.e. Touma \& Palme (2005), and Schwarzenberger (2007).

For the development of techniques for fecal steroid analysis experiments on the metabolism of radioactively labeled steroids have provided insight into the metabolism and the excretion of hormone metabolites via feces and urine (Palme et al., 2005). The route of excretion varies considerably among species, and between steroids within the same species. Results affirmed that estrogens in the form of estradiol and/or estrone are present in fecal samples and as such can easily be determined by using specific assay or a total estrogen assay. In contrast, testosterone, progesterone, and especially cortisol/ corticosterone are heavily metabolized and the original hormone is barely present in the feces (Schwarzenberger et al., 1996; Möstl \& Plame, 2002; Palme et al., 2005; Touma \& Palme, 2005). Therefore specific assays i.e. those typically used for the analysis of hormones in blood samples are of limited value and should be replaced by assay with high cross-reactions against a certain group of steroids, i.e. group-specific assays (Schwarzenberger et al., 1997; Palme et al., 2005). A well proven approach is to test several such assays when validating a system for a given species.

Because of species specific differences in hormone metabolite excretion, validation should be done separately for even closely related species (Graham, 2004; Keay et al., 2006; Millspaugh \& Washburn, 2004; Möstl \& Palme, 2002; Palme et al., 2005; Schwarzenberger et al., 1997; Touma $\&$ Palme, 2005). Validation in reproductive studies is rather straight forward as progesterone metabolites fluctuate with the estrous cycle, however, validation studying the stress response is more difficult and usually is based on an ACTH challenge test (Möstl \& Palme, 2002; Palme et al., 2005; Touma \& Palme, 2005). In addition to 
metabolism and hence the use of an appropriate immunoassay with adequate cross-reactivities, other concerns in fecal steroid analysis are extraction techniques, storage and stability of fecal metabolites, determination of fecal immunoreactive steroid metabolites by HPLC, gut transit time, diurnal and seasonal variations, as well as gender and diet. As currently researchers worldwide are applying different techniques, results between studies are only comparable in their physiologic outcome, but usually not in absolute metabolite concentrations (Graham, 2004; Keay et al., 2006; Millspaugh \& Washburn, 2004; Möstl \& Palme, 2002; Palme et al., 2005; Schwarzenberger et al., 1996, 1997, 2007; Touma \& Palme, 2005).

In recent years a series of comprehensive studies using fecal steroid analysis have been applied for studying female and male reproductive physiology, i.e. estrous cycle length, spontaneous $v s$. induced ovulation, pregnancy, lactational anovulation, age at the onset of puberty, seasonal patterns of reproduction. Fecal steroid analysis has been used to assess various fertility control techniques i.e. ultrasonographic monitoring of ovarian events and fetal gestational parameters, contraceptive treatments, estrous synchronization, endocrine responses to ovulation induction protocols. Another important field of application is the study of the activities of the hypothalamicpituitary-adrenal (HPA) axis and how this is modulated in response to stress, i.e. in relation to behaviour, social status, population density, dominance rank, territoriality, reproduction, environmental factors, disturbance, as an indicator of well-being and welfare, pain, presence of predators, parasites, and as a predictor of mortality.

Further details on this topic are available from a series of comprehensive reviews published recently by myself (the author of this abstract), and by my colleagues Dr. R. Palme and Dr. E. Möstl. Due to copyright restriction granted for the most recent reviews this extended abstract is providing essential and important facts for fecal steroid analysis, but it does not repeat recently published material in full details. However, I am happy to provide any of the cited reviews upon request directed to my above mentioned e-mail address.

\section{Literature cited}

BROWN, J.L. Comparative endocrinology of domestic and nondomestic felids. Theriogenology, v.66, p.25-36, 2006.

GRAHAM, L.H. Non-invasive monitoring of reproduction in zoo and wildlife species. Annual Review of Biomedical Sciences, v.6, p.91-98, 2004.

KEAY, J.M.; SINGH, J.; GAUNT, M.C. et al. Fecal glucocorticoids and their metabolites as indicators of stress in various mammalian species: A literature review. Journal of Zoo and Wildlife Medicine, v.37, p.234-244, 2006.

MILLSPAUGH, J.J.; WASHBURN, B.E. Use of fecal glucocorticoid metabolite measures in conservation biology research: Considerations for application and interpretation. General and Comparative Endocrinology, v.138, p.189199, 2004

MÖSTL, E.; PALME, R. Hormones as indicators of stress. Domestic Animal Endocrinology, v.23, p.67-74, 2002.

PALME, R.; RETTENBACHER, S.; TOUMA, C. et al. Stress hormones in mammals and birds: Comparative aspects regarding metabolism, excretion, and noninvasive measurement in fecal samples. Annals of the New York Academy of Sciences v.1040, p.162-171, 2005.

ROTH, T.L. A review of the reproductive physiology of rhinoceros species in captivity. International Zoo Yearbook, v.40, p.130-143, 2006.

SCHWARZENBERGER, F. The many uses of non-invasive faecal steroid monitoring in zoo and wildlife species. International Zoo Yearbook, in press, 2007.

SCHWARZENBERGER, F.; MÖSTL, E.; PALME, R. et al.Faecal steroid analysis for non-invasive monitoring of reproductive status in farm, wild and zoo animals. Animal Reproduction Science, v.42, p.515-526, 1996.

SCHWARZENBERGER, F; PALME, R.; BAMBERG, E. et al. A review of faecal progesterone metabolite analysis for non-invasive monitoring of reproductive function in mammals. Zeitschrift für Säugetierkunde, v.62, Suppl. 2, p.214-221, 1997.

SHIMIZU, K. Studies on Reproductive Endocrinology in Nonhuman Primates: Application of Non-invasive Methods. Journal of Reproduction and Development, v.51, p.1-13, 2005.

TOUMA, C.; PALME, R. Measuring fecal glucocorticoid metabolites in mammals and birds: The importance of validation. Annals of the New York Academy of Sciences, 